\title{
Radio Detection of Supernova 2004ip in the Circumnuclear Region of the Luminous Infrared Galaxy IRAS 18293-3413
}

\author{
M.A. Pérez-Torres \\ Instituto de Astrofísica de Andalucia, IAA-CSIC, Apdo. 3004, 18080 Granada, Spain, \\ torres@iaa.es \\ S. Mattila ${ }^{1}$ \\ Tuorla Observatory, University of Turku, Väisäläntie 20, FI-21500 Piikkiö, Finland, \\ S.Mattila@qub.ac.uk \\ A. Alberdi \\ Instituto de Astrofísica de Andalucia, IAA-CSIC, Apdo. 3004, 18080 Granada, Spain, \\ antxon@iaa.es \\ L. Colina \\ Instituto de Estructura de la Materia, IEM-CSIC, 28006 Madrid, Spain, \\ colina@damir.iem.csic.es \\ J.M. Torrelles \\ Instituto de Ciencias del Espacio (CSIC)-IEEC, Facultat de Física, Universitat de \\ Barcelona, Martí i Franquès 1, 08028 Barcelona, Spain, \\ torrelles@ieec.fcr.es \\ P. Väisänen \\ South African Astronomical Observatory, PO Box 9, Observatory 7935, South Africa, \\ petri@saao.ac.za \\ S. Ryder \\ Anglo-Australian Observatory, PO Box 296, Epping, NSW 1710, Australia, \\ sdr@aao.gov.au \\ N. Panagia ${ }^{2,3}$ \\ STScI, Baltimore, MD 21218, USA, \\ panagia@stsci.edu
}

A. Wilson 
Astronomy Department, Univ. of Maryland, College Park, MD 20742, USA, wilson@astro.umd.edu

\begin{abstract}
We report a radio detection of supernova SN 2004ip in the circumnuclear region of the luminous infrared galaxy IRAS 18293-3413, using Very Large Array (VLA) observations at $8.4 \mathrm{GHz}$ on 11 June 2007. SN 2004ip had been previously discovered at near-infrared wavelengths using adaptive optics observations, but its nature (core-collapse or thermonuclear) could not be definitely established. Our radio detection, about three years after the explosion of the supernova, indicates a prominent interaction of the ejecta of SN 2004ip with the circumstellar medium, confirming that the supernova was a core-collapse event (probably a Type II), and strongly suggesting that its progenitor was a massive star with a significant mass-loss prior to its explosion. SN 2004ip has a $8.4 \mathrm{GHz}$ luminosity of $3.5 \times 10^{27} \mathrm{erg} \mathrm{s}^{-1} \mathrm{~Hz}^{-1}$, about three times as bright as SN $2000 \mathrm{ft}$ in NGC 7469 at a similar age, and given its projected distance to the nucleus $(\sim 500 \mathrm{pc})$, is one of the closest of all known radio SNe to a galaxy nucleus, and one of the brightest radio $\mathrm{SNe}$ ever.
\end{abstract}

Subject headings: — galaxies: starburst — supernovae: individual (SN 2004ip)

- radio continuum: stars — radio continuum: galaxies

\title{
1. Introduction
}

Stars more massive than $\sim 8 \mathrm{M}_{\odot}$ explode as core-collapse supernovae (CCSNe), i.e., Types Ib/c and II. Given a reasonable assumption for the initial mass function (IMF), the observed CCSN rate can be used as a direct measure of the current star formation rate (SFR). Thus, CCSN searches (e.g. Cappellaro et al. 1999; Dahlen et al. 2004; Cappellaro et al. 2005) can yield a new independent measurement of the star formation history of the Universe. However, optical searches are only able to discover the SNe not severely affected

\footnotetext{
${ }^{1}$ Astrophysics Research Centre, School of Mathematics and Physics, Queen's University Belfast, BT7 $1 \mathrm{NN}, \mathrm{UK}$.

${ }^{2}$ INAF-CT, Osservatorio Astrofisico di Catania, I-95123 Catania, Italy.

${ }^{3}$ Supernova Ltd. OYV \# 131, Virgin Gorda, British Virgin Islands.
} 
by dust extinction and therefore most of the SNe occurring in dusty starburst galaxies have remained undiscovered even in the local Universe.

Furthermore, a large fraction of the star formation at high- $z$ took place in luminous $\left(\mathrm{L}_{\mathrm{IR}}>10^{11} \mathrm{~L}_{\odot} ;\right.$ LIRGs $)$ and ultraluminous $\left(\mathrm{L}_{\mathrm{IR}}>10^{12} \mathrm{~L}_{\odot}\right.$; ULIRGs $)$ infrared galaxies (Pérez-González et al. 2005) where the dust extinction is even more severe. The existence of hidden SN factories in the nuclear and circumnuclear regions of (U)LIRGs has already been demonstrated by high-resolution radio observations. For example, VLA observations of the circumnuclear starburst in NGC 7469 revealed an extremely bright radio supernova, SN 2000ft (Colina et al. 2001b). More recently, very long baseline interferometry (VLBI) observations of the nearby ULIRG, Arp 220, have revealed luminous radio SNe within its innermost $150 \mathrm{pc}$ nuclear regions at a rate of $4 \pm 2 \mathrm{yr}^{-1}$, which indicates a SFR high enough to power its entire IR luminosity (Lonsdale et al. 2006). These studies confirm that CCSN rates a couple of orders of magnitude higher than in ordinary field galaxies can be expected for starburst dominated (U)LIRGs.

Ground-based near-infrared (IR) observations (with spatial resolution $\sim 1$ arcsec) can provide a complementary tool to high-resolution radio observations in the search for CCSNe in (U)LIRGs (Van Buren et al. 1994; Grossan et al. 1999; Mattila \& Meikle 2001; Maiolino et al. 2002; Mannucci et al. 2003; Mattila et al. 2004; Mattila et al. 2005a,b). More recently, Mattila et al. (2007a,b) reported the first-ever adaptive optics (AO) assisted discovery of a SN making use of the NAOS CONICA (NACO) AO system on the ESO Very Large Telescope (VLT). As a result of their $\mathrm{K}_{\mathrm{S}}$-band [2.2 microns] search for highly-obscured CCSNe in a sample of (U)LIRGs (see also Väisänen et al. 2007), SN 2004ip was discovered in IRAS 18293-3413, a LIRG at a distance of $79 \mathrm{Mpc}$ (at this distance 1 arcsecond corresponds to 383 pc), with an estimated host galaxy extinction towards SN 2004ip between 5 and 40 magnitudes in $A_{\mathrm{V}}$ (Mattila et al. 2007b). SN 2004ip is located in the nuclear regions of IRAS 18293-3413 at 1".14 east and 0".78 north of (about 500 pc projected distance from) the galaxy's $\mathrm{K}_{\mathrm{s}}$-band nucleus.

It has been proposed that CCSNe exploding in dense environments, like those encountered in the circumnuclear regions of starburst galaxies, can result in very luminous radio SNe (Chevalier 1982, Chugai 1997). An apparent confirmation of such a theory came from the discovery of the radio bright SN 2000ft (Colina et al. 2001a,b) in a circumnuclear starburst ring ( $~ 600$ pc from the nucleus) of its LIRG host NGC 7469 ( $D \approx 70 \mathrm{Mpc}$ ). However, the case of SN 2000ft is outstanding because its six-year long VLA monitoring has shown that the supernova shares essentially the same properties that are common to radio SNe identified as Type II SNe, despite having exploded in the dusty and very dense environment of the circumnuclear region (Alberdi et al. 2006). We also note that the optical glow of SN 
2000ft has recently been identified in archival HST images taken on 13 May 2000 (Colina et al. 2007), consistent with the predicted date of the explosion from the analysis of its radio light curves (Alberdi et al. 2006).

The observations of SN 2004ip at near-IR wavelengths did not allow it to be unambiguously identified with either a core-collapse (Type Ib/c, or II SN), or thermonuclear (Type Ia) event. Furthermore, the discovery of SN 2004ip could only be announced very recently (Mattila et al. 2007a), thus making any follow-up observations difficult. However, assuming that SN 2004ip was a SN 2000ft-like event, we could expect it to be bright enough as to be detectable with the VLA even three years after its explosion. Hence, we proposed rapid response science VLA time to search for cm-wavelength radio emission at its location. In this Letter, we report the first radio detection of SN 2004ip, indicating a strong interaction with its circumstellar medium.

\section{VLA Observations}

We observed the host galaxy of SN 2004ip, IRAS 18293-3413, on 11 June 2007 at the frequency of $8.4 \mathrm{GHz}$ with the VLA in A configuration, aimed at resolving and detecting SN 2004ip at radio wavelengths. We used rapid response exploratory VLA Time, since the angular distance of the supernova to the nucleus (about 1.4 arcsec) made it necessary to ask for the VLA in its most extended, A-configuration. We observed at $8.4 \mathrm{GHz}$, which resulted in an angular resolution of $0^{\prime \prime}$.62 and 0.21 in right ascension (R.A.) and declination (decl.), respectively, enough to discern the radio emission from the supernova from that of the nucleus. Also, at $8.4 \mathrm{GHz}$ any extended radio emission from the galaxy and its nucleus should be less prominent than at lower frequencies (the only previously existing VLA radio observations of IRAS 18293-3413 were carried-out at $1.4 \mathrm{GHz}$ with an angular resolution of about 5 arcseconds, indicating a large flux density, $\sim 130 \mathrm{mJy}$ for the entire galaxy), while at higher frequencies the supernova was expected to be too faint to be detectable.

The observations lasted for two hours, and consisted of $~ 11.5$ min scans on SN 2004ip (for a total on-target time of $\sim 81 \mathrm{~min}$ ), interleaved with $\sim 2.5 \mathrm{~min}$ scans on the phase and amplitude calibrator J1820-254, and each time ending with a $\sim 5$ min observation of the quasar 3C $286(1331+305)$ to set the absolute flux density scale. We edited, calibrated, and imaged our $8.4 \mathrm{GHz}$ VLA data by following standard data reduction techniques implemented within the NRAO Astronomical Image Processing System (AIPS). 


\section{Results}

Our results are summarized in Figure 1 and Table 1. Figure 1 shows the $8.4 \mathrm{GHz}$ radio emission from the central parts of IRAS 18293-3413 (contours) overlaid on a subtracted NACO $\mathrm{K}_{\mathrm{S}}$-band image (shown with an inverted brightness scale), which is the result of subtracting a $\mathrm{K}_{\mathrm{S}}$-band image obtained on 4th May 2004 from the image obtained on 15th September 2004 (for details see Mattila et al. 2007b). A strong negative residual coincident with the $\mathrm{K}_{\mathrm{S}}$-band nucleus is visible in white color, and SN 2004ip is clearly visible as a positive point source (in black colour). A local maximum of radio emission within the circumnuclear region of the galaxy is right coincident with SN 2004ip.

The radio contours also indicate evidence for a number of bright, compact objects whose discussion is beyond the scope of this Letter. We only note here that we have found eight such compact regions with signal-to-noise ratios larger than $20\left(S_{\nu} \gtrsim 460 \mu \mathrm{Jy}\right)$, but which

have no clear counterpart in the near-IR image. Future multi-wavelength VLA observations of this galaxy will allow us to shed some light on the nature of these compact sources. In Table 1, we show the 8.4 GHz VLA flux densities of the nucleus of IRAS 18293-3413 and a source we identify as SN 2004ip, which exploded between 4th May and 13th September 2004 within the nuclear starburst of the galaxy.

The absolute position of SN 2004ip was only reported with an estimated precision of \pm 0.4 " by Mattila et al. (2007b). To compare with the position of the source detected in our VLA image (see Fig. 1), the precision of the supernova astrometry first needed to be improved. For this purpose we used archival K-band data of IRAS 18293-3413 obtained with the SOFI near-IR camera on the New Technology Telescope (NTT) on 8th September 2001. The final K-band image, reduced using standard IRAF routines, has an on-source exposure time of $30 \mathrm{~min}$ and a seeing FWHM of $\sim 1.2$ ". Due to the low galactic latitude a large number of stars is visible within the $\sim 7 " \times 7 "$ field of view of the combined NTT image. Therefore, we were able to identify over 400 bright and isolated stars with astrometry available from 2MASS. These yielded a world coordinate system (WCS) solution for the NTT image with rms of 4 and 3 milliarcsec (mas) in R.A. and Decl., respectively. We then used the centroid coordinates of 14 isolated stars, detected within the field of view of both the 42 " $\times 42$ " NACO and NTT images, to align the images in IRAF using a general geometric transformation with no non-linear part. This yielded an rms of 34 and 22 mas in R.A. and Decl., respectively. Finally, the position of the supernova was measured in a NACO $\mathrm{K}_{\mathrm{S}}$-band image which is the result of subtracting an image obtained on 4th May 2004 from an image obtained on 15th September 2004 (for details on the images and the subtraction method see Mattila et al. 2007b). We adopted the average from three different methods (centroid, gauss, ofilter) used in IRAF as the SN position. The position uncertainty as indicated by the 
standard deviation of these measurements was very small $\sim 1$ mas for both $x$ and $y$, thanks to the well sampled NACO PSF and the flat and close to zero background in the subtracted image. Using the WCS of the NTT image aligned to the NACO image, we obtained R.A. $=18 \mathrm{~h} 32 \mathrm{~m} 41.207 \mathrm{~s}$ and Decl. $=-34^{\circ} 11^{\prime} 26.80^{\prime \prime}$ (equinox 2000.0) for the SN with an estimated precision of \pm 34 and \pm 22 mas in R.A. and Decl., respectively.

Therefore, the radio source we identified as SN 2004ip appears coincident with the nearIR position of the supernova within 10 and 20 mas in R.A. and Decl., respectively. This is within the uncertainties of the SN near-IR position (as derived above) and radio position ( \pm 20 mas and \pm 10 mas in R.A. and Decl., respectively), which confirms that the radio emission corresponds to SN 2004ip.

\section{Discussion and Summary}

Thermonuclear (Type Ia) supernovae are not expected to be strong radio emitters, and have not yet been detected at radio wavelengths (e.g., Panagia et al. 2006). Current modelling of their radio emission indicates that the circumstellar wind around the progenitor star is much less dense than in the case of CCSNe, and would be overrun in about one day due to its proximity and the much higher velocity of the supernova blast wave. At the distance of IRAS 18293-3413, this would result in an SN Ia radio emission reaching $8.4 \mathrm{GHz}$ peak values of (13-50) $\mu \mathrm{Jy}$ at around (3-10) days after the explosion, and quickly decreasing below (0.3-3) $\mu$ Jy after a hundred days, depending on the particular model (P. Lundqvist, private communication). Therefore, a thermonuclear origin for SN 2004ip -detected at $\sim 470 \mu \mathrm{Jy}$ after more than 1000 days after its explosion- can be ruled out.

CCSNe are expected - as opposed to thermonuclear SNe- to become strong radio emitters when the SN ejecta interact with the circumstellar medium (CSM) that was ejected by the progenitor star before its explosion as a supernova (Chevalier 1982; Weiler et al. 1986). Indeed, the interaction gives rise to a high-energy density shell, which is Rayleigh-Taylor unstable and can drive turbulent motions that may amplify the existing magnetic field and efficiently accelerate relativistic electrons, thus enhancing the emission of synchrotron radiation at radio wavelengths (Chevalier 1982). The duration of this radio SN phase is limited, however, by the extent of the expanding wind of the progenitor star, which can reach a radius where the ram pressure of the wind, $\rho_{w} v_{w}^{2}$, equals the external pressure of the insterstellar medium ISM, $P_{I S M}$ (Chevalier \& Fransson 2001). For a spherically symmetric, steady wind $\left(\rho_{w} \propto r^{-2}\right)$, this radius is $r_{w} \approx 0.18 \dot{M}_{-4}^{1 / 2} v_{w 1}^{1 / 2} p_{7}^{-1 / 2} \mathrm{pc}$, where $\dot{M}_{-4}$ is the mass loss rate in units of $10^{-4} \mathrm{M}_{\odot} \mathrm{yr}^{-1}, v_{w 1}$ is the wind velocity in units of $10 \mathrm{~km} \mathrm{~s}^{-1}$, and $p_{7}$ is the ISM pressure in units of $10^{7} \mathrm{~cm}^{-3} \mathrm{~K}$, which is the estimated pressure for the central 
region of the starburst in M 82 (Chevalier \& Clegg 1985). At this distance, a CCSN would then enter the supernova remnant (SNR) phase, and the radio emission would no longer be due to the interaction with the CSM, but rather with the ISM. However, even if the ejecta of SN 2004ip would have been freely expanding at a constant velocity of $v_{s}=10^{4} \mathrm{~km} \mathrm{~s}^{-1}$, the distance reached by the circumstellar shock in three years would be $r \lesssim 0.03$ pc, which is much smaller than $r_{w}$ assuming an ISM pressure similar to M 82. We conclude that SN 2004ip is still in its SN phase and its radio emission is being powered -even three years after the SN explosion- by prominent interaction with the CSM. This fact confirms that the supernova was a core-collapse event, and implies that its progenitor was a massive star with a significant mass-loss $\left(\dot{M} \gtrsim 10^{-4} \mathrm{M}_{\odot} \mathrm{yr}^{-1}\right)$ prior to its core collapse.

SN 2004ip exploded sometime between 4th May and 13th September 2004, so its radio emission is now likely in a decaying phase. The $8.4 \mathrm{GHz}$ flux density of SN 2004ip detected in the June 2007 observations $(\sim 470 \mu \mathrm{Jy})$ correspond to an isotropic luminosity of $3.5 \times 10^{27} \mathrm{erg} \mathrm{s}^{-1} \mathrm{~Hz}^{-1}$, or three times as luminous as SN 2000ft in NGC 7469 at a similar age. Given the fact that it exploded about three years earlier, SN 2004ip might have been one of the most luminous radio SNe ever at its peak (and probably significantly brighter than the nucleus of its host galaxy), and would therefore belong to the class of extremely bright and long-lasting radio SNe, like SN 1978K (Schlegel et al. 1999), SN 1986J (Weiler et al. 1990; Pérez-Torres et al. 2002), SN 1988Z (Van Dyk et al. 1993b), or SN $2000 \mathrm{ft}$ (Colina et al. 2001b), which at their peak emission were a thousand to a few thousand times more luminous than Cas A, the brightest radio SN in the Milky Way (Weiler et al. 1986). In addition to its extreme radio brightness, SN 2004ip shares with SN 2000ft in the LIRG NGC 7469 (Alberdi et al. 2006), and with the young radio supernovae in the central regions of Arp 299 (Neff et al. 2004), other characteristics in common. Indeed, SN 2004ip exploded in the circumnuclear region of a Luminous Infrared Galaxy, is located at a similar projected distance $(r \sim 500 \mathrm{pc})$ from the galaxy nucleus, is still in its radio SN phase, and is detectable several years after its explosion, These facts indicate that such radio SNe might be a relatively common phenomenon in circumnuclear starburst environments.

Extremely bright and long lasting radio SNe are identified in the optical as Type II supernovae, and because of their huge radio luminosities, their progenitors are believed to be massive stars in the 20 to $30 \mathrm{M}_{\odot}$ range (Weiler et al. 1990; Van Dyk et al. 1993) that explode in very dense environments (e. g., Chugai 1997). The typical spectral index for normal Type II supernovae is -0.6 to -0.8 (Weiler et al. 2002). Type II radio SNe also exhibit slow rises and declines, and normally take more than one year to reach their $8.4 \mathrm{GHz}$ peak radio emission. On the other hand, Type Ib/c supernovae can also produce bright radio SNe, e.g., SN 1983N (Sramek et al. 1984; Weiler et al. 1986) and SN 1990B (van Dyk et al. 1993a), and the brightest of all, SN 1998bw (Kulkarni et al. 1998). However, the radio 
emission of these supernovae is characterized by a rather fast rise and decay (few days to a few weeks), and by radio spectral indices of about -1.1 to -1.2 (Weiler et al. 2002). While the measured single radio flux of SN 2004ip does not allow a definite classification, the fact that its radio luminosity is so high even three years after the explosion favours strongly a Type II origin since their radio flux densities tend to decrease much more slowly than the radio emission from Type $\mathrm{Ib} / \mathrm{c}$ SNe.

The level of the $8.4 \mathrm{GHz}$ radio emission of SN 2004ip detected in our June 2007 observation $(\sim 470 \mu \mathrm{Jy})$ suggests that we might be able to monitor the radio flux density evolution of SN 2004ip with the VLA for some years from now, thus probing the circumstellar interaction around the supernova, eventually allowing us to detect its transition from the SN phase to the SNR phase, as has been suggested for a number of compact sources in the nuclear starburst of Arp 220 (Parra et al. 2007). In addition, a radio light curve follow-up could provide more detailed information on the progenitor of SN 2004ip. For example, Ryder et al. (2004) inferred the action of a binary companion from periodic modulations in the radio light curve of SN 2000ig, which was subsequently confirmed from imaging at Gemini (Ryder et al. 2006). This would make SN 2004ip the second case, only after SN 2000ft in NGC 7469, where such radio monitoring has been carried out for a SN in the circumnuclear starburst of a LIRG, and will help to better understand the behaviour of SNe within dense starburst environments (e.g., Alberdi et al. 2006). Furthermore, Mattila et al. (2007b) estimated an average SFR of $135 \mathrm{M}_{\odot} \mathrm{yr}^{-1}$ for IRAS 18293-3413 corresponding to a CCSN rate of about 1.0 SN per year. Therefore, radio monitoring at high-resolution and sensitivity with the VLA might easily result also in the discovery of new radio SNe.

The number of CCSNe discovered in circumnuclear regions of (U)LIRGs, both at infrared and radio wavelengths, is still small. However, these events may have an important impact when estimating the complete local CCSN rates including also the SNe in the optically obscured parts of the galaxies. The direct detection and study of CCSNe in starburst galaxies over a large range of IR luminosities $\left(\mathrm{L}_{\mathrm{IR}}=10^{10}-10^{12} \mathrm{~L}_{\odot}\right)$ is also crucial for interpreting the results of the high-z CCSN searches, since a large fraction of the massive star formation at high- $z$ took place in IR luminous galaxies. Therefore, our discovery shows that the combination of high-resolution observations at near-infrared and radio wavelengths is a powerful tool to search for CCSN events from LIRGs in the local Universe, and thus establish their core-collapse supernova rates and star formation rates, as well as to constrain the nature of the discovered events through their interaction with the surrounding medium.

We are grateful to an anonymous referee for helpful comments on the manuscript. We are also grateful to the National Radio Astronomy Observatory (NRAO) for granting us Rapid Response, Very Large Array (VLA) time for this project. NRAO is a facility of the National 
Science Foundation operated under cooperative agreement by Associated Universities Inc. MAPT research is supported by the Ramón y Cajal programme of the Spanish Spanish Ministry of Education and Science. MAPT and AA, and JMT acknowledge support from the Spanish grants AYA2006-14986-C02-C01 and AYA2005-08523-C03, respectively. SM acknowledges financial support from the Participating Organisations of EURYI and the EC Sixth Framework Programme and from the Academy of Finland (project: 8120503).

\section{REFERENCES}

Alberdi, A., Colina, L., Torrelles, J.M., Panagia, N., Wilson, A.S., \& Garrington, S. 2006, ApJ 638, 938

Cappellaro, E., Evans, R., Turatto, M. 1999, A\&A, 351, 459

Cappellaro, E., et al. 2005, A\&A, 430, 83

Chevalier, R. A. 1982, ApJ, 259, 302

Chevalier, R. A., \& Clegg, A. W. 1985, Nature, 317, 44

Chevalier, R. A., \& Fransson, C. 2001, ApJL, 558, L27

Chugai, N. N. 1997, Astronomy Reports, 41, 672

Colina, L., Alberdi, A., Torrelles, J. M., Panagia, N., \& Wilson, A. S. 2001a, IAU Circ., 7587,1

Colina, L., Alberdi, A., Torrelles, J. M., Panagia, N., \& Wilson, A. S. 2001b, ApJL, 553, L19

Colina, L., Díaz-Santos, T., Alonso-Herrero, A., Panagia, N., Alberdi, A., Torrelles, J. M., \& Wilson, A. S. 2007, A\&A, 467, 559

Dahlen, T., et al. 2004, ApJ, 613, 189

Grossan, B., Spillar, E., Tripp, R., Pirzkal, N., Sutin, B. M., Johnson, P., \& Barnaby, D. 1999, AJ, 118, 705

Kulkarni, S. R., et al. 1998, Nature, 395, 663

Lonsdale, C. J., Diamond, P. J., Thrall, H., Smith, H. E., \& Lonsdale, C. J. 2006, ApJ, 647, 185 
Maiolino, R., Vanzi, L., Mannucci, F. et al. 2002, A\&A, 389, 84

Mannucci, F., et al. 2003, A\&A, 401, 519

Mattila S. \& Meikle W.P.S. 2001, MNRAS, 324, 325

Mattila, S., Meikle, W. P. S., \& Greimel R. 2004, New Astronomy Review, 48, 595

Mattila, S., Greimel, R., Gerardy, C., \& Meikle, W. P. S 2005a, IAUC, 8474, 1

Mattila, S., et al. 2005b, IAUC, 8473, 1

Mattila, S., et al. 2007a, Central Bureau Electronic Telegrams, 858, 1

Mattila, S., et al. 2007b, ApJ, 659, L9

Neff, S. G., Ulvestad, J. S., \& Teng, S. H. 2004, ApJ, 611, 186

Panagia, N., Van Dyk, S. D., Weiler, K. W., Sramek, R. A., Stockdale, C. J., \& Murata, K. P. 2006, ApJ, 646, 369

Parra, R., Conway, J. E., Diamond, P. J., Thrall, H., Lonsdale, C. J., Lonsdale, C. J., \& Smith, H. E. 2007, ApJ, 659, 314

Pérez-González, P. G., et al. 2005, ApJ, 630, 82

Pérez-Torres, M. A., Alberdi, A., Marcaide, J. M., Guirado, J. C., Lara, L., Mantovani, F., Ros, E., \& Weiler, K. W. 2002, MNRAS, 335, L23

Ryder, S. D., Sadler, E. M., Subrahmanyan, R., Weiler, K. W., Panagia, N., \& Stockdale, C. 2004, MNRAS, 349, 1093

Ryder, S. D., Murrowood, C. E., \& Stathakis, R. A. 2006, MNRAS, 369, L32

Schlegel, E. M., Ryder, S., Staveley-Smith, L., Petre, R., Colbert, E., Dopita, M., \& Campbell-Wilson, D. 1999, AJ, 118, 2689

Sramek, R.A., Panagia, N., \& Weiler, K.W. 1984, ApJ 285, L59

Vaisanen, P., et al. 2007, ArXiv e-prints, 708, arXiv:0708.2365

Van Buren, D. \& Greenhouse, M.A. 1994, ApJ, 431, 640

Van Dyk, S. D., Sramek, R. A., Weiler, K. W., \& Panagia, N. 1993a, ApJ, 409, 162

van Dyk, S. D., Weiler, K. W., Sramek, R. A., \& Panagia, N. 1993b, ApJ, 419, L69 
Weiler, K. W., Sramek, R. A., Panagia, N., van der Hulst, J. M., \& Salvati, M. 1986, ApJ, 301,790

Weiler, K.W., Panagia, N., \& Sramek, R.A. 1990, ApJ 364, 611

Weiler, K. W., Panagia, N., Montes, M. J., \& Sramek, R. A. 2002, ARA\&A, 40, 387 
Table 1. $8.4 \mathrm{GHz}$ VLA positions and flux densities of SN 2004ip and the nucleus of its host galaxy IRAS 18293-3413

\begin{tabular}{lccr}
\hline \hline Component & $\alpha(\mathrm{J} 2000)$ & $\delta(\mathrm{J} 2000)$ & \multicolumn{1}{c}{$\mathrm{S}_{\nu}[\mu \mathrm{Jy}]$} \\
\hline SN 2004ip & $18^{\mathrm{h}} 32^{\mathrm{m}} 41^{\mathrm{s}} .208$ & $-34^{\circ} 11^{\prime} 26^{\prime \prime} .82$ & $466 \pm 25$ \\
Nucleus & $18^{\mathrm{h}} 32^{\mathrm{m}} 41^{\mathrm{s}} .108$ & $-34^{\circ} 11^{\prime} 27^{\prime \prime} .62$ & $1708 \pm 41$ \\
\hline
\end{tabular}

Note. - The errors in the flux density measurements represent one statistical standard deviation, $\sigma_{f}$, and are calculated as $\sigma_{f}^{2}=\left(\epsilon S_{0}\right)^{2}+\sigma_{0}^{2}$, where $S_{0}$ is the measured peak flux density, $\sigma_{0}$ is the off-source rms, and the fractional error $\epsilon$ (assumed to be of $2 \%$ ) accounts for the inaccuracy of VLA flux density calibration and possible deviations of the primary calibrator from an absolute flux density scale. 


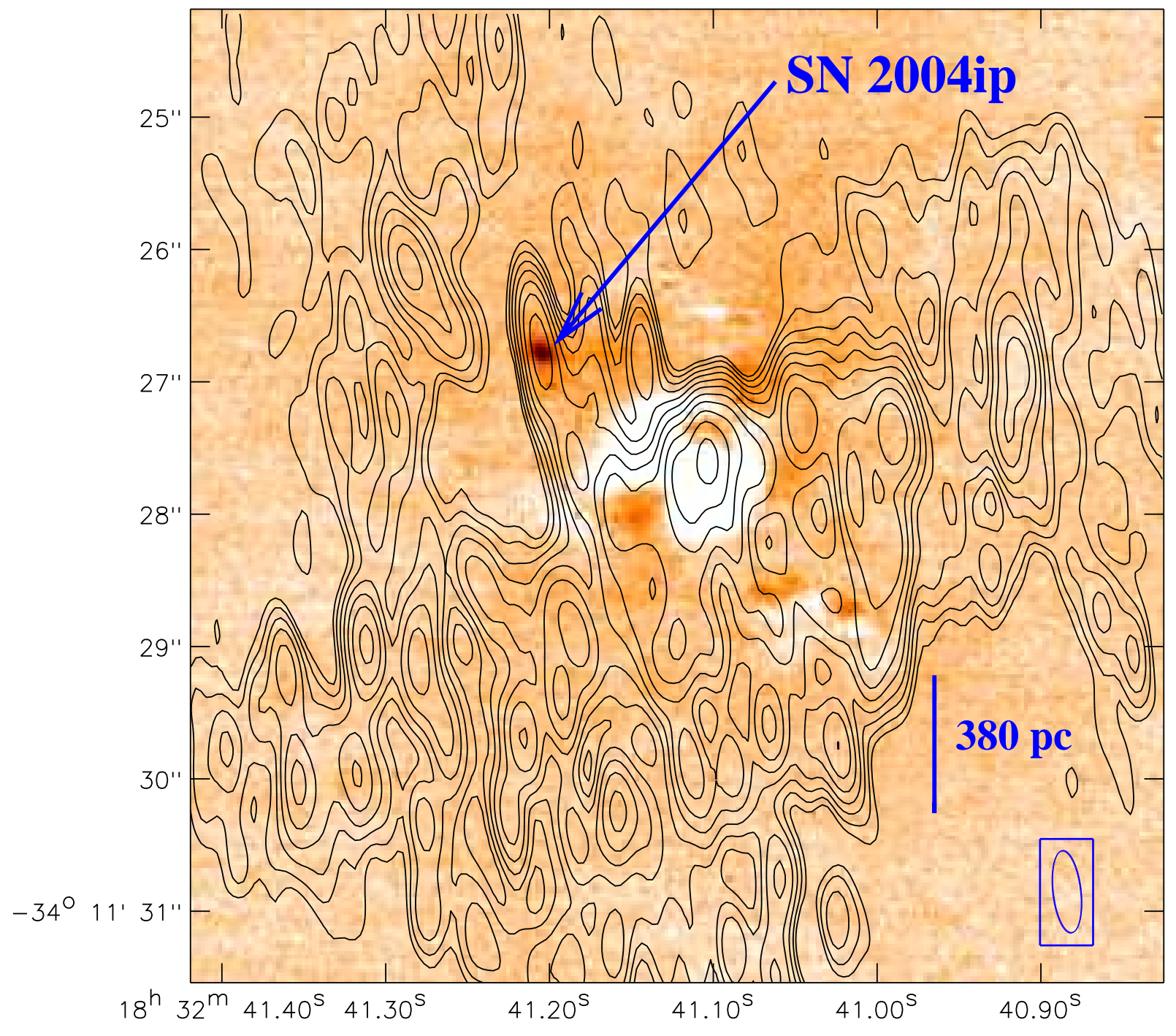

Fig. 1.- Contours of $8.4 \mathrm{GHz}$ observations of IRAS 18293-3413 made on 11 June 2007 with the VLA in A configuration, overlaid on a NACO $\mathrm{K}_{\mathrm{S}}$-band (near-IR) image (shown with an inverted brightness scale). A local maximum of radio emission within the circumnuclear region of the galaxy is coincident with SN 2004ip. The VLA contours are drawn at ($2,2,2 \sqrt{2}, 4, \ldots) \times$ the off-source rms noise in the image, which is $23 \mu \mathrm{Jy}_{\text {beam }}{ }^{-1}$, and the synthesized beam size is of $0^{\prime \prime} 62 \times 00^{\prime \prime} 21$ along P.A. 6.5. The $8.4 \mathrm{GHz}$ peak brightness is $1.71 \mathrm{mJy}$ beam $^{-1}$, and corresponds to the radio nucleus of IRAS 18293-3413. The total continuum $8.4 \mathrm{GHz}$ emission from IRAS $18293-3413$ above $3 \sigma$ is $40.1 \mathrm{mJy}$. 